\title{
Effect of misalignment on rod lip seal behaviour
}

\author{
B. Pinedo ${ }^{1}$, M. Conte ${ }^{1}$, J. Aguirrebeitia ${ }^{2} \&$ A. Igartua ${ }^{1}$ \\ ${ }^{1}$ Ik4- Tekniker, Tribology Unit, Eibar, Spain \\ ${ }^{2}$ ETSI-BILBAO, Department of Mechanical Engineering, \\ University of Basque Country, Bilbao, Spain
}

\begin{abstract}
The most common failure mechanism of pneumatic cylinders is related to wear of head seals. It usually occurs due to excessive radial loads or vibrations (dynamic run-out) or as consequence of an incorrect mounting (rod to bore misalignment). Sealing behaviour has been the object of several analytical and numerical studies orientated to reproduce actual working conditions but perfect rod alignment has been often considered.

A tri-dimensional quasi-static numerical model of rod radial misalignment and its effect on facing seal behaviour is herein presented as a first step of a study concerning mounting effects on head seal performances. In the specific, a thermoplastic polyurethane (TPU) rod lip seal has been considered. An analytical model was also developed considering the characteristics of the rubber-like material and stiffness variation of the lip seal versus deformation. A non-linear dependence of stiffness on deformation of rubber-like material was found. A cross validation between analytical and numerical models is provided.

Keywords: lip seal, rod misalignment, eccentricity, stiffness.
\end{abstract}

\section{Introduction}

Seals are critical components for many mechanical devices such as pneumatic and hydraulic actuators, valves, pumps and engines. Several studies on sealing systems resulted in a wide variety of seal geometries and materials, and a better knowledge about seal behaviour during operation. However, due to the wide range of involved parameters every sealing operation is a challenge. Geometry of the cross section, material and roughness of seals and relative counterparts, 
sliding speed, sealed fluid properties and manufacturing tolerances of the system affect leakage rate, friction and wear of sealing components. Design and study of sealing systems require modelling and a deep knowledge of material properties especially if polymers are involved. Conte et al. demonstrated firstly the influence of fillers on tribological behaviour of polymer composites [1] and then the influence of frictional heating was analyzed considering their thermal characteristics $[2,3]$.

Development of numerical and experimental techniques, advanced finite elements modelling tools and the increasing knowledge concerning polymers behaviour in the last years, allow us to deal with modelling of many complex phenomena taking place at the sealing surface. Lubrication models $[4,7-10]$ and roughness models $[5,6]$ of elastomeric seals were developed in order to predict seal behaviour and calculate leakage rates under different operating conditions, with a subsequent better understanding of the seal performances.

Aim of this study was the development of a modelling procedure for estimating the effects of rod radial misalignment on a sealing system. In fact, although mounting eccentricity of rods is a very common cause of failure of seals, its effect on the seal service life has not been studied enough. Some authors studied the relation between rod eccentricity and the leakage rate of rotatory rod lip seals for different speed regimes. Mokhtar et al. [11] experimentally proved that an increase of shaft radial eccentricity results in higher leakage rates whereas an increase of shaft angular misalignment results in lower leakage rates. Besides, results showed that for a specific shaft misalignment, an increase of shaft rotating speed results in lower leakage rates, however, increasing the fluid pressure the opposite effect is achieved. Amabili et al. [12] found that there is a relationship between shaft rotating speed and dynamic eccentricity. Results showed that for any eccentricity the leakage rate increases with the shaft rotating speed until a maximum is reached from which the leakage rate decreases with a speed increase. The lower the dynamic eccentricity the higher the speed at which this maximum leakage rate takes place. Conte [13] analytically studied necessary fluid pressure to prevent fluid leakage when a rod angular eccentricity exists, modelling a rod lip seal in 2D by means of a torsional spring of known stiffness. Leakage rate as a function of fluid pressure was also study in this work.

Although there are few studies about the influence of rod eccentricity on seals life, in the case of ball bearings, many authors have already studied the effect of a wrong bearing alignment on its behaviour: Amasorrain et al. [14] studied resulting axial and radial forces and the total moment in four contact-point slewing bearing as a function of a combination of radial, axial and angular bearing misalignment, and Aguirrebeitia et al. [15] studied radial, axial and tilting-moment load combinations resulting in a static failure also in four contactpoint slewing bearings.

During the last decades, many authors have dealt with the problem of expressing seal contact force as a function of seal section compression level. Most of these studies were focused on elastomeric O-rings. Lindley $[16,17]$ offered a non-dimensional force-deflection relationship for an O-ring based on 
Hertz theory. This relationship was composed by a term derived analytically assuming plain strain conditions and another correction term for high compression levels of the seal. Green and English [18] presented general expressions of hyperelastic O-ring type seal stiffness based on numerical data and compared results with those estimated by Lindley. They also found that the differences between the results obtained from axisymmetric and plain strain loading conditions increase with the seal compression level. Some years later, Hyung-Kyu Kim et al. [19] analyzed elastomeric O-ring contact forces under compression, comparing Lindley's analytical formula results with numerical and experimental results obtained by means of an opportune test rig. They found that results by Lindley's formula were closer to numerical results than to experimental ones. They also found that numerical results underestimated contact forces under a fractional compression of $24 \%$ and overestimated them for frictional compressions of $32 \%$. So far, no exact analytical model of the contact force variation as a function of compression level has been found. The main cause of difficulties to reproduce the contact force of seals regards elastomeric materials behaviour, that is, variable elastic modulus, Poisson ratio closed to 0.5 and stress relaxation phenomenon. In this work, a whole lip seal contact force is analytically and numerically studied as a function of the rod radial position.

The main objective of this paper is to obtain an analytical $3 \mathrm{D}$ rod radial misalignment model of a thermoplastic polyurethane (TPU) reciprocating lip seal. A static case has been analyzed and absence of any fluid. The model is based on the characterization of the seal as an ensemble of springs subjected to traction/compression. The analytical model is completed with a combination of simulation results of bidimensional and tridimensional Finite Element models.

\section{Method}

A numerical tridimensional model has been developed in Ansys environment to study the effect of rod misalignment on the resultant sealing force at the contact surface. An analytical model of a rod lip seal has been developed considering geometrical interference as function of rod radial eccentricity. The contact force between the seal cross section and the rod as a function of interference was also considered. The thermoplastic polyurethane (TPU) has a non-linear behaviour as illustrated in Figure 1 which shows a typical compressive stress-strain curve of

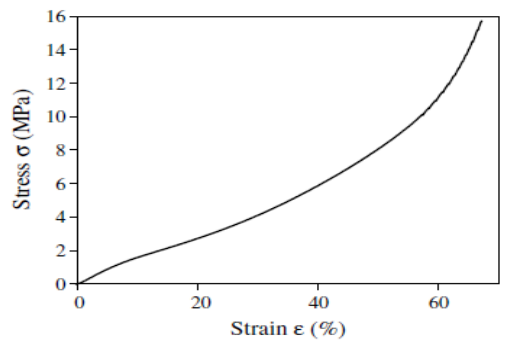

Figure 1: $\quad$ Compressive stress-strain curve of TPU [20]. 
the considered material [20]. The proposed analytical model has been completed and adjusted by means of numerical results. Figure 2 shows a scheme of the proposed process for obtaining an analytical rod misalignment model of a seal.

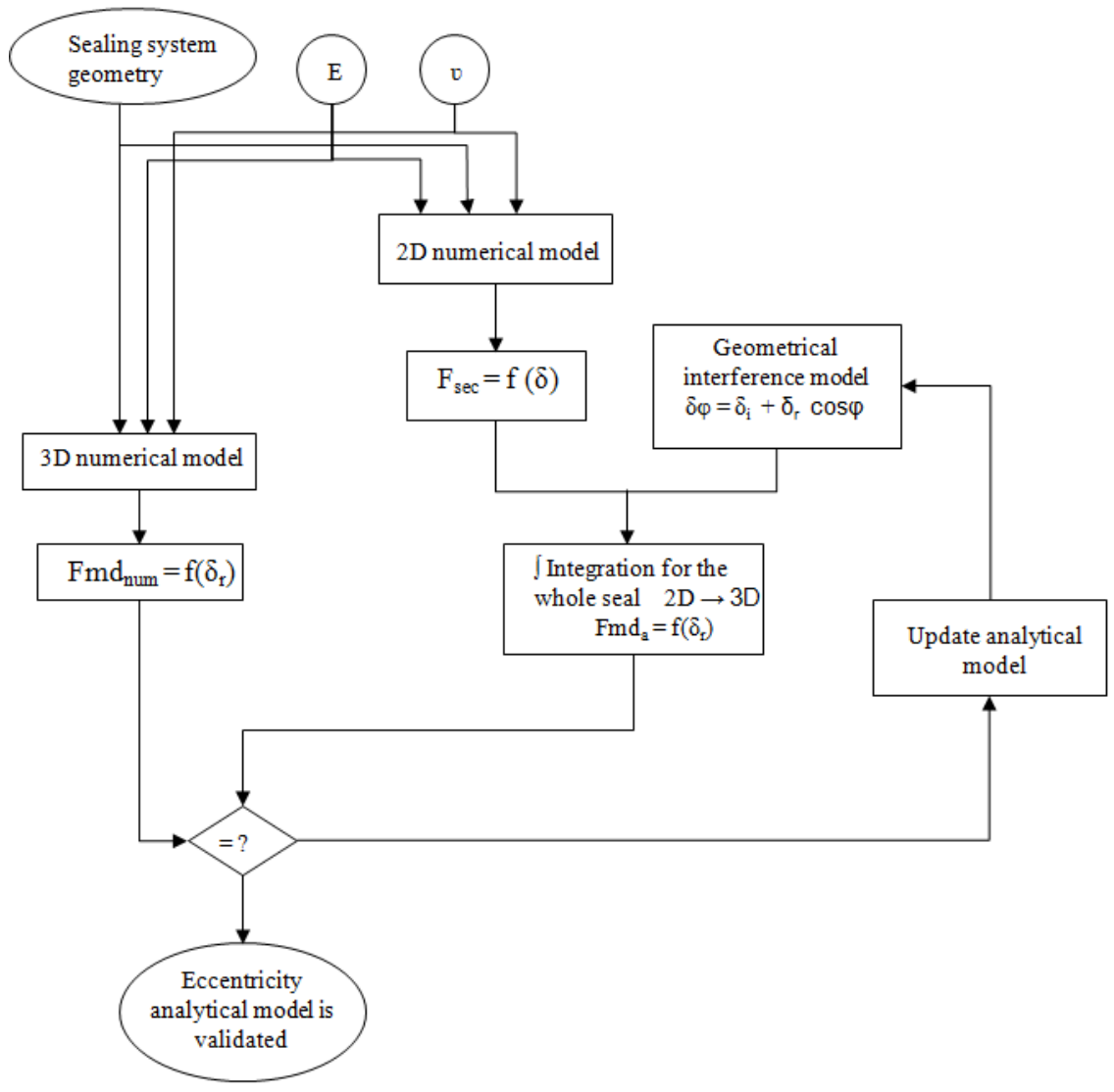

Figure 2: Proposed process scheme.

\subsection{Numerical model}

A numerical study was performed to complete the analytical model described in detail in the next section. For this purpose, a commercial finite element software was used. Both, a plane model and a tridimensional model of the lip rod seal were developed using quadrilateral and triangular elements.

The tridimensional model was built to estimate the rod lip seal behaviour as a function of rod misalignment. Reaction force resultants of the tridimensional analysis are mandatory inputs to complete the analytical model.

The plane model was built in order to obtain the functional contact relation between the rod and the seal as a function of seal section interference, necessary to build the analytical model. 
The 3D lip seal model was built by means of 171911 quadrilateral and triangular elements. In the bidimensional model, the lip seal was modelled by means of Plane183 solid elements, with a total of 2278 elements. Combin14 spring type elements were added to the $2 \mathrm{D}$ model in order to limit the free rotation of the lip seal section. Figure 3 shows the generated mesh in both cases.
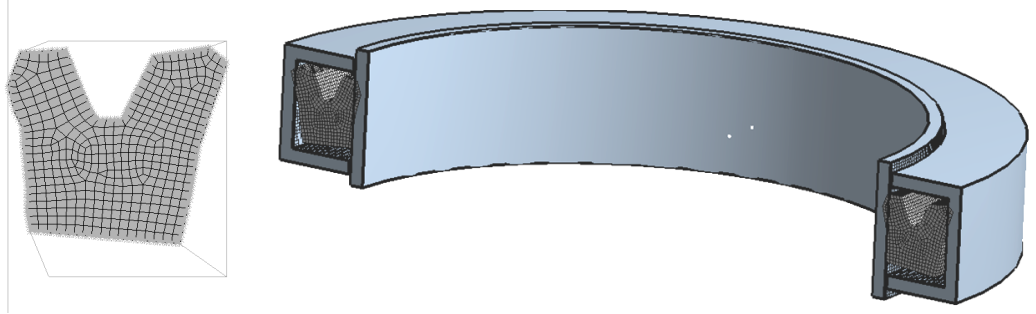

Figure 3: Bidimensional (left) and tridimensional (right) models of the sealing system.

In both models a node to surface contact was chosen to simulate both the seal/rod and seal/groove contact. Boundary conditions take into account friction at contact surfaces. The friction coefficients were set to 0.1 for the seal/rod contact and 0.4 for the seal/groove contact, in case of dry contact [21].

As a first approximation, the seal material, TPU, was considered an incompressible, isotropic and linear elastic material since for strain levels up to $20 \%$, the maximum seal strain, stress-strain behaviour can be considered linear (Figure 1). Young modulus and Poisson's ratio were assumed to be equal to $12 \mathrm{MPa}$ and 0.499 , respectively, according to manufacturer communication. The rod and the bore have been considered rigid elements since polymer stiffness is much lower than steel stiffness.

Boundary conditions allowed rod displacement just in the selected radial misalignment direction. Rod displacement was applied step by step, from an initial position simulating ideal mounting conditions where the rod and bore were perfectly centered to the maximum allowed displacement, determined by the gap between the rod and the cylinder inner diameter. The cylinder bore was considered perfectly static.

\subsection{Analytical model}

Elastomeric seals are constrained in grooves and compressed between the 7 and $30 \%$ [22]. This compression, which is also described as interference, squeeze or nip, is usually perpendicular to the rod. Selection of the correct amount of interference is important.

The development of the analytical model is based on the assumption of an ideal initial situation where the seal is mounted in the rod completely centered. In this situation, the interference is the same along the whole seal, $\delta_{i}$. From this ideal situation, a radial misalignment $\delta_{r}$ is applied to the rod in order to study a common real situation and its effects on seal behaviour. When the rod is 
displaced, contact characteristics will change over the whole seal. Figure 4 shows the geometrical interference between the rod and the seal for a specific misalignment of $\delta_{r}$. The angle $\varphi$ is a polar coordinate that defines the position of each section relative to the rod radial misalignment direction. Therefore, a geometrical-interference model is formulated so that the geometrical-interference field as a function of the misalignment can be expressed as in eqn. (1),

$$
\delta_{\varphi}=\delta_{i}+\delta_{r} \cos \varphi
$$

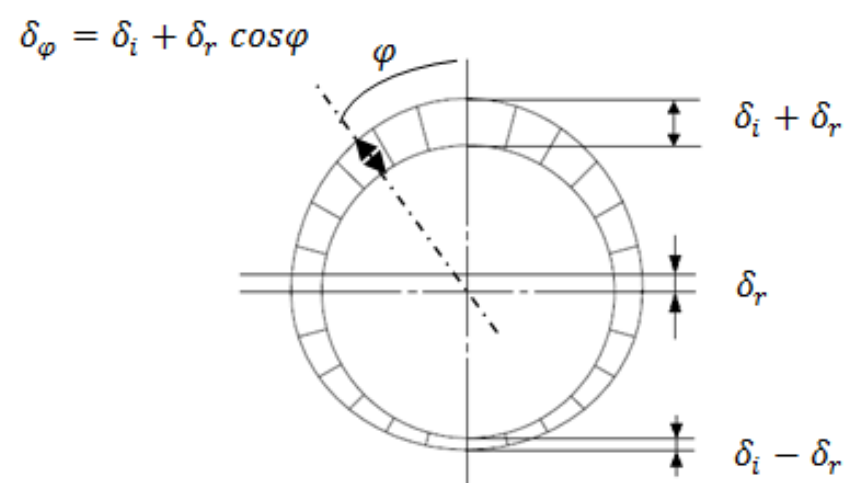

Figure 4: Geometric interference model.

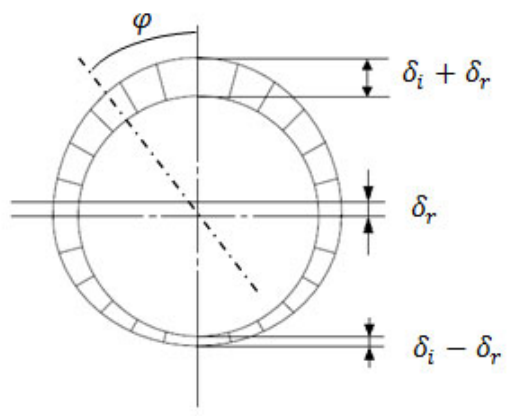

$$
\begin{gathered}
\delta_{i}>0 \\
\delta_{i}>\delta_{r} \\
-\pi \leq \varphi \leq \pi
\end{gathered}
$$

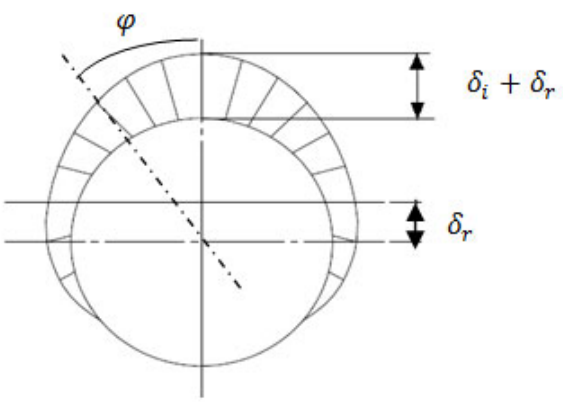

$$
\begin{aligned}
& \delta_{i}>0 \\
& \delta_{i}>\delta_{r} \\
& -a \cos \left(-\frac{\delta_{i}}{\delta_{r}}\right) \leq \varphi \leq \operatorname{acos}\left(\frac{\delta_{i}}{\delta_{r}}\right)
\end{aligned}
$$

Figure 5: Possible interference configuration as function of the value of radial misalignment. 
The applicable maximum misalignment and concentric position seal/counterparts interference values are determined by manufacturing tolerances. Depending on the relation between concentric position interference value and rod misalignment value, it is possible to distinguish two different cases. Figure 5 shows the possible interference configurations. If the rod misalignment value is lower than the seal/counterparts interference in a concentric position, all the sections in the seal will keep in contact with the rod. Otherwise, the contact will be lost in a specific area of the seal.

The contact force between a seal section and the rod depends on its compression grade and it can be defined by a functional contact relation, which for a specific sealing system can be expressed as,

$$
F_{\text {sec }}=f\left(\delta_{\varphi}\right)
$$

So the radial contact force in a differential section of the seal is,

$$
\frac{d F_{s e c}}{r_{m} d \varphi}=f\left(\delta_{\varphi}\right)
$$

Each seal section/rod contact force in the misalignment direction can be expressed as follows,

$$
d F r_{m d}=d F_{\text {sec }} * \cos \varphi
$$

As a result, eqn. (3) can be written as,

$$
d F r_{m d}=r_{m} f\left(\delta_{\varphi}\right) \cos \varphi d \varphi
$$

The resultant load in the dynamic lip seal can be calculated integrating eqn. (5) for the whole seal circumference. If the interference of the seal in the concentric position, $\delta_{i}$, is bigger than the rod radial misalignment, $\delta_{r}$, the total load supported by the dynamic lip of the seal in the misalignment direction can be expressed in eqn. (6).

$$
F r_{m d}=2 r_{m} \int_{0}^{\pi} f\left(\delta_{\varphi}\right) \cos \varphi d \varphi
$$

Eqn. (7) expresses the total contact force in the misalignment direction when seal interference in the concentric position is lower than the radial rod misalignment.

$$
F r_{m d}=2 r_{m} \int_{0}^{\operatorname{arcos}\left(-\delta_{i} / \delta_{r}\right)} f\left(\delta_{\varphi}\right) \cos \varphi d \varphi
$$

The average seal section radius $r_{m}$ is measured from the rod center in a concentric position. In this situation, the value of the average radius is in every section the same, $r_{i}$. When the rod is misaligned, the average radius in each section can be expressed as,

$$
r_{m}=r_{i}+\delta_{r} \cos \varphi
$$

In all the cases, the maximum admissible rod misalignment is determined by design tolerances. 


\section{Results and discussion}

Firstly, the tridimensional model of the sealing system was built in order to observe the effect of rod eccentricity on the seal stress and strain fields and on the contact force between the seal and the rod. Rod misalignment simulations were carried out step by step starting from a concentric ideal position of the rod up to the maximum applicable misalignment. The maximum applicable misalignment is determined by the gap $e$ between the rod and the bore $(0.2 \mathrm{~mm}$ according to manufacturing drawing). Results showed a non-linear relation between the resultant contact force in the dynamic lip of the seal, $F r_{m d}$, and the rod radial misalignment, i.e., the seal showed a variable stiffness with the rod position. The whole seal stiffness in the misalignment direction decreases with a rod eccentricity increase as can be observed in Figure 6.

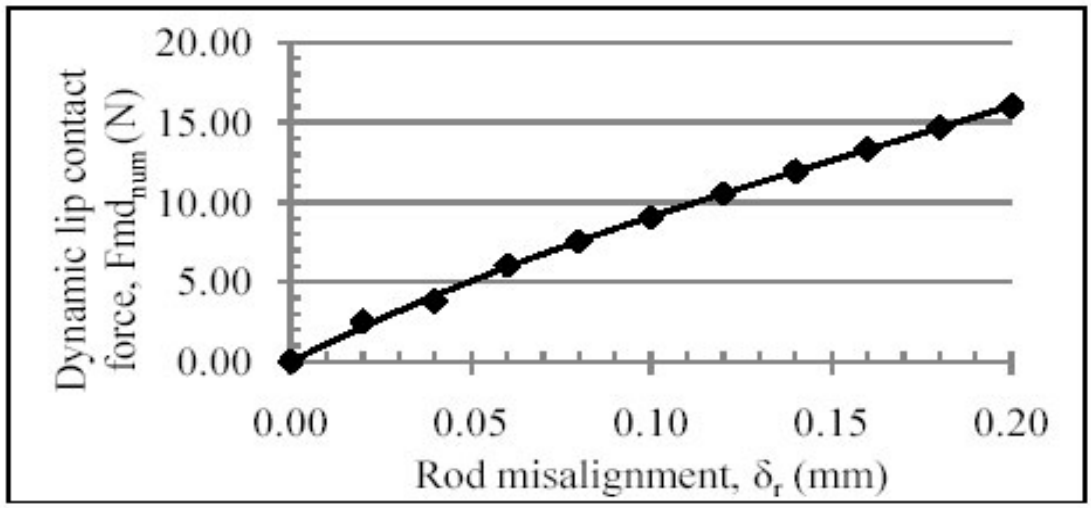

Figure 6: Numerical results of the dynamic lip resultant contact force, $\boldsymbol{F r}_{\boldsymbol{m d}}$, as a function of rod misalignment, $\boldsymbol{\delta}_{\boldsymbol{r}}$.

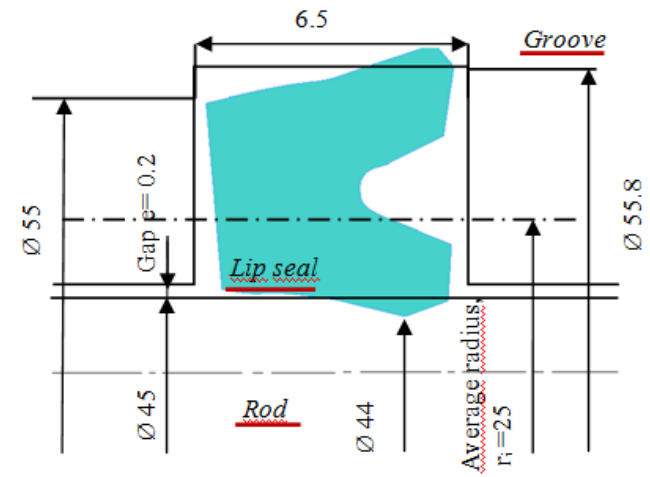

Figure 7: $\quad$ Principal dimensions of the studied seal geometry (in mm). 
Plain strain simulations were carried out in order to develop an analytical model reproducing the lip seal behaviour, numerically estimated as a function of rod misalignment. Figure 7 shows the lip seal studied in this work. As initial mounting interference, $\delta_{i}$, it was adopted a value of $0.9 \mathrm{~mm}$ according to design tolerances. Hence, the interference configuration studied in this work corresponds to the $\delta_{i}>\delta_{r}$ one. Plain strain simulation results are shown in Figure 8. A variable stiffness of the studied lip seal section as a function of compression was found. Analytically, the functional contact relation between the rod and the lip seal section depending on the interference or compression can be expressed as,

$$
F_{\text {sec }}=-0.5474 \delta_{\varphi}{ }^{3}+1.4109 \delta_{\varphi}{ }^{2}-0.1557 \delta_{\varphi}
$$

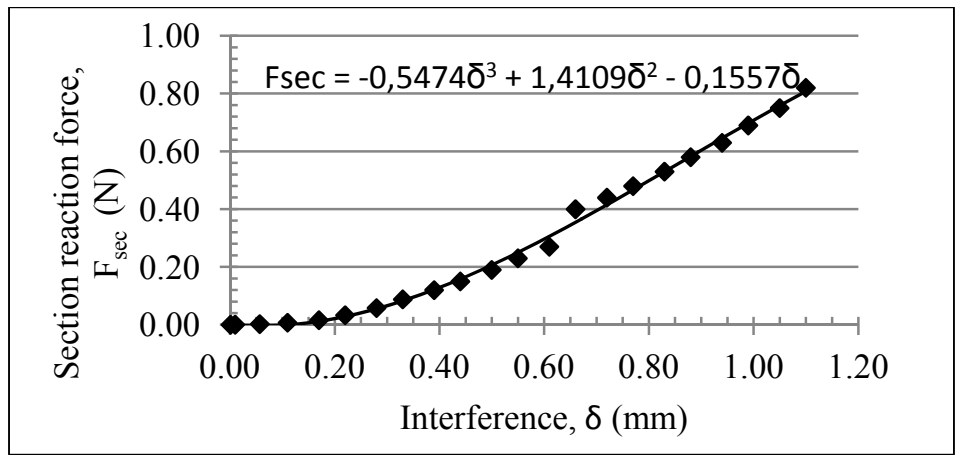

Figure 8: Reaction force of the dynamic lip of the seal section as a function of the interference or compression.

Substituting the geometrical interference model defined by eqn. (1) and integrating results for the whole seal as described in section 2.2, the contact force of the whole seal for any rod eccentricity can be calculated. Eqn. (10) expresses the contact force in the rod as a function of rod misalignment.

$$
\mathrm{Fmd}_{a}=83.84 \delta_{r}[\mathrm{~N}]
$$

Figure 9 shows a comparison between the analytical misalignment model and numerically obtained results. A good matching was found. However, the analytical solution results in constant seal stiffness while the numerical one does not. The maximum difference between the analytical and numerical results in absolute terms is approximately of $0.85 \mathrm{~N}$ and it appears for a rod misalignment value of $0.06 \mathrm{~mm}$. Figure 9 also presents the error between analytical and numerical results in percentage terms for rod misalignments up to the maximum admissible. The maximum percentage error appears for very low eccentricity values of about $0.02 \mathrm{~mm}$ and the lowest error values are for the highest admissible eccentricity values, i.e., numerical and analytical result matching is better for high rod eccentricity values. 


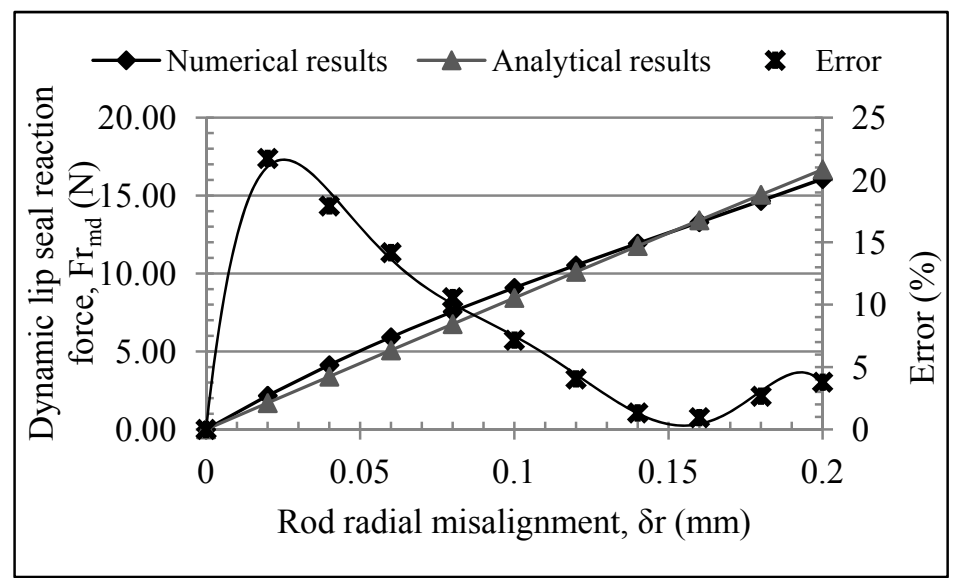

Figure 9: Comparison between numerical and analytical results.

Plain strain and tridimensional simulations contact force results for a concentric position of the rod and the bore are very similar, so the problem does not seem to be caused by numerical modelling. It is thought that the main cause of stiffness difference in both models is that the analytical model does not take into account many factors, such as, seal torsional stiffness, seal deformation effects, and any tangential effect and their variation as a function of rod eccentricity.

In order to add to the rod misalignment model those tridimensional effects missed in the contact force integration based on a bidimensional model, the analytical model represented by eqn. (10) can be updated by means of numerically obtained results as follows,

$$
\mathrm{Fmd}_{a}=83.84 \delta_{r}+\left(649.94 \delta_{r}^{3}-290.47 \delta_{r}^{2}+29.05 \delta_{r}\right)
$$

The modelling procedure developed in this work for estimating the effects of rod radial misalignment on a sealing system by means of contact forces, ended in similar results to numerically obtained ones. Nevertheless, the procedure should be optimized including tangential, torsional and deformation effects that allow us to reproduce seal behaviour when a mounting eccentricity exists.

\section{References}

[1] Conte M., Fuentes E., Fernandez X., Igartua A., Effect of fillers on tribological properties of PTFE materials. Ecotrib 2011, June 2011, Wien, Austria, pp. 207-212.

[2] Conte M., Fernandez B., Igartua A., Effect of surface temperature on tribological behavior of PTFE composites. 10th International Conference on Surface Effects and Contact Mechanics, September 2012, Malta.

[3] Conte M. Fernandez B., Igartua A. Frictional heating calculation based on tailored experimental tests. NordTrib 2012, Trondheim, Norway. 
[4] Salant R. F. Modelling rotary lip seals. Wear 207 (1997) 92-99.

[5] Salant R. F., Thatte A., Transient EHL analysis of an elastomeric hydraulic seal. Trib. Int. 42 (2009) 1424-1432.

[6] Salant R. F., Shen D., An unsteady mixed soft EHL model, with application to a rotary lip seal. Trib. Int. 40 (2007) 646-651.

[7] Nikas G. K., Sayles R. S. Nonlinear elasticity of rectangular elastomeric seals and its effects on elastohydrodynamic numerical analysis. Tribology International 37 (2004) 651-660.

[8] Nikas George K. Theoretical study of solid back-up rings for elastomeric seals in hydraulic actuators. Trib. Int. 37 (2004) 689-699.

[9] Nikas G.K., Sayles R. S. Computational model of tandem rectangular elastomeric seals for reciprocating motion. Trib. Int. 39 (2006) 622-634.

[10] Nikas G.K., Sayles R. S. Study of leakage and friction of flexible seals for steady motion via a numerical approximation motion. Trib. Int. 39 (2006) 921-936.

[11] Mokhtar M. O. A., Mohamed M.A.A., El-Giddawy M.E., Yassen S.A.Y. On the effect of misalignment on the performance of U-type lip seal. Wear 223 (1998) 139-142.

[12] Amabili M., Colombo G., Prati E. Leakage of radial lip seals at large dynamic eccentricities. Proceedings of the 16th international Conference on Fluid Sealing, September 18-20, Brugge, Belgium. (2000) 321-333.

[13] Conte M. MSc Thesis. Capitolo 3: Modello di guarnizione a labbro.

[14] Amasorrain J.I., Sagartzazu X., Damián J. Load distribution in a four contact-point slewing bearing. Mechanism and Machine Theory 38 (2003) 479-496.

[15] Aguirrebeitia J., Abasolo M., Avilés R., Fernandez de Bustos I., Calculation of General Static Load-Carrying Capacity for the Design of Four-Contact-Point Slewing Bearings. ASME Journal of Mechanical Design, 132 (6), 064501 (6 pages).

[16] Lindley, P. B., Load-Compression Relationships of Rubber Units. Jour. Of Strain Analysis, 1, (1966) 190-195.

[17] Lindley, P. B., Compression Characteristics of Laterally Unrestrained Rubber O-ring. Journal of International Rubber Inst., 1, (1967) 202-213.

[18] Green I., English C., Analysis of Elastomeric O-ring Seals in Compression Using the Finite Element Method. Tribology Transactions, 1, (1992) 83-88.

[19] Hyung-Kyu Kim, Sung-Han Park, Hyan-Gyu Lee, Dong-Ryun Kim, Young-Ho Lee. Approximation of contact stress for a compressed and laterally one side restrained O-ring. Engineering Failure Analysis 14. (2007) 1680-1692.

[20] Elleuch R., Elleuch K., Salah B., Zahouani H. Tribological behaviour of thermoplastic polyurethane elastomers. Materials and Design 28 (2007) 824-830.

[21] Conte M. Interaction between seals and counterparts in pneumatic and hydraulic components. Thesis. Politecnico di Torino. (2008).

[22] Flitney Robert. Seals and sealing handbook. $5^{\text {th }}$ edition. Elsevier. (2007). 